\title{
Investigation of the stresses in continuous thin films and patterned lines by $x$-ray diffraction
}

\author{
W.-M. Kuschke and E. Arzt \\ Max-Planck-Institut für Metallforschung, Seestr. 92, 70174 Stuttgart, Germany
}

(Received 27 September 1993; accepted for publication 6 December 1993)

\begin{abstract}
Strains and stresses in aluminum thin films and patterned lines were measured using $x$-ray diffraction. Measurements were performed on pure aluminum and on ion-implanted aluminum, as annealed and six months after an annealing treatment. The results suggest that stresses in passivated lines, starting from an unequitriaxial state of stress, show the tendency to relax in the direction of an equitriaxial state of stress, depending on the ratio of grain size and linewidth or film thickness. The relaxation is particularly rapid in ion-implanted aluminum lines, in contradiction to the expected strengthening effect. Possible implications for electromigration resistance are discussed.
\end{abstract}

Because of the mismatch in the thermal expansion coefficients of aluminum and silicon large thermal stresses can arise in thin $\mathrm{Al}$ films and lines on Si substrates during cooling from some elevated temperature, e.g. ${ }^{1}$ These stresses can, at least partially, be relaxed by plastic yielding and by stress induccd void formation. ${ }^{2}$ The remaining stresses, are a measure for the stress the material can endure without deformation or damage, a quantity which has been suggested to correlate with electromigration resistance of conductor lines. ${ }^{3}$ With this aim in mind, the stresses in continuous and patterned films have been analyzed by $x$-ray diffraction.

$X$-ray diffraction was done by measuring the interplanar spacing of a particular set of crystallographic planes, namely the (422) planes, using different incident angles $\alpha$. The angle $\alpha$ is related to the inclination angle $\Psi$, defined as the angle $\Psi=\Theta-\alpha$ between the film surface and the plane investigated, with $\Theta$ being half the scattering angle $2 \Theta$. Plotting $d(\Psi)$ vs $\sin ^{2} \Psi$ and fitting the data points with a straight line yields, by extrapolation of $d(\Psi)$ to $\Psi=0^{\circ}$ and $\Psi=90^{\circ}$, the interplanar distances of planes parallel and perpendicular to the film surface, respectively. ${ }^{4}$ Using the unstressed lattice spacing $d_{0}$, these $d$ values can be converted to strains according to $\varepsilon=\left(d-d_{0}\right) / d_{0}$.

In the case of lines two $d(\Psi)$ measurements have to be done. One measurement was performed along the length of the lines and another along the width, leading to the distance of planes perpendicular to the film surface in the length $\left(d_{x}\right)$ and width $\left(d_{y}\right)$ directions of the lines, respectively. The spacing of planes parallel to the film surface $d_{z}$ can be taken from both measurements. In principle both measurements should yield the same value for $d_{z}$, i.e., the straight lines in the $d(\Psi)$ vs $\sin ^{2} \Psi$ plot of both measurements should intersect at $\sin ^{2} \Psi=0$. Besides the scatter of the measured $d(\Psi)$ values around the fitted straight line, the difference between the $d_{z}$ values of both measurements gives a good cstimatc for the quality of the measurement.

Stresses were calculated from strains by using the stiffness matrix. This requires a transformation of the stiffness tensor from the [100], [010], [001] crystallographic axis system to the sample coordinate system which corresponds to a (111) fiber texture, as is usually found in thin $\mathrm{Al}$ films on silicon. Due to this texture $d_{0}$ cannot be measured directly. It is found by an interpolation in the $d(\Psi)$ vs $\sin ^{2} \Psi$ plot at $\sin ^{2} \Psi_{0}=2 C_{13}^{\prime} /\left(2 C_{13}^{\prime}+C_{33}^{\prime}\right)$. The $C_{i j}^{\prime}$ are stiffness coefficients in the sample coordinate system. They are functions of $C_{11}, C_{12}$, and $C_{44}$, defined in the crystallographic coordinate system. ${ }^{4}$

X-ray diffraction was performed using a Siemens D5000 powder diffractometer with $\mathrm{Cu} K \alpha$ radiation. Measurements were made directly after an annealing treatment of $1 \mathrm{~h}$ at $450{ }^{\circ} \mathrm{C}$ in forming gas (specimens denoted by as annealed) and six months later when most of the relaxation had taken place (denoted by relaxed). The samples were from the same processing lot as those studied in Ref. 5, i.e., 0.5- $\mu \mathrm{m}$-thick aluminum films (AL) on thermally oxidized silicon, with some samples being oxygen-ion implanted (II) in order to produce a strengthening effect. Grain sizes were $1.4 \mu \mathrm{m}$ for $\mathrm{AL}$ and $0.35 \mu \mathrm{m}$ for II. For the stress measurement on lines, an area of $8 \times 16 \mathrm{~mm}^{2}$ was patterned to $1.5-\mu \mathrm{m}$-wide lines separated by $1.5-\mu \mathrm{m}$-wide spacings which corresponds to nearly 2700 parallel lines. In some AL and II samples the effect of a passivation was studied by depositing an low thermal oxide layer of $0.5-\mu \mathrm{m}$ thickness at $350{ }^{\circ} \mathrm{C}$.

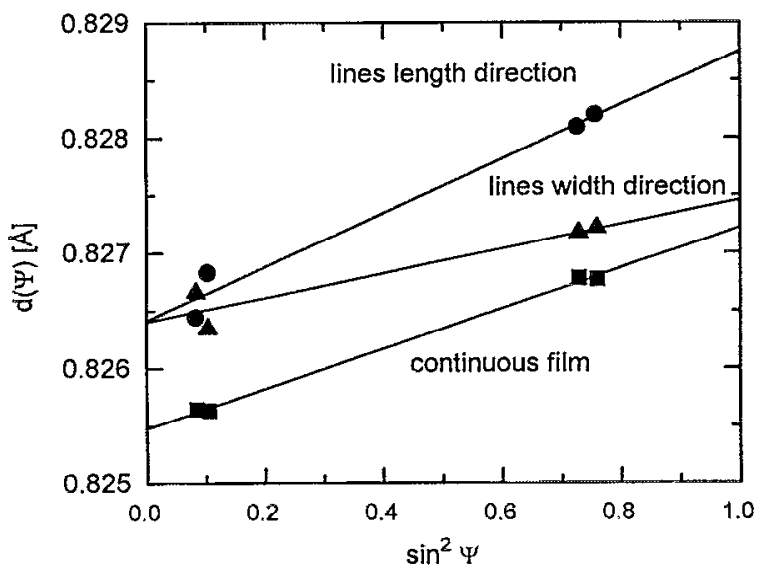

FIG. 1. $d(\Psi)$ vs $\sin ^{2} \Psi$ plot for passivated aluminum continuous thin film and patterned lines after relaxation. 
TABLE I. Summary of the strains (in units of $10^{-3}$ ) measured in continuous films and patterned lines.

\begin{tabular}{|c|c|c|c|c|c|c|}
\hline & & \multicolumn{2}{|c|}{ Films } & \multicolumn{3}{|c|}{ Lines } \\
\hline \multicolumn{2}{|c|}{ Sample description } & $\epsilon_{x, y}$ & $\epsilon_{z}$ & $\epsilon_{x}$ & $\epsilon_{y}$ & $\epsilon_{z}$ \\
\hline $\mathrm{AL}$ & as annealcd & 1.75 & -1.78 & 3.91 & -0.35 & -1.62 \\
\hline (unpassivated) & relaxed & 1.23 & -1.25 & 1.63 & -0.15 & -0.92 \\
\hline $\mathrm{AL}$ & as annealed & $1.40_{-}$ & -1.42 & 4.31 & 3.63 & -2.33 \\
\hline (passivated) & relaxed & 0.92 & -0.94 & 2.76 & 1.20 & -0.07 \\
\hline II & as annealed & 3.86 & -3.92 & 2.75 & 0.04 & -1.18 \\
\hline (unpassivated) & relaxed & 1.49 & -1.51 & 2.01 & 0.12 & -0.89 \\
\hline II & as annealed & 4.12 & -4.19 & 3.30 & 1.73 & -1.07 \\
\hline (passivated) & relaxed & 2.19 & -2.23 & 1.09 & 0.81 & 0.49 \\
\hline
\end{tabular}

Figure 1 shows a typical $d(\Psi)$ vs $\sin ^{2} \Psi$ plot of the unpassivated AL continuous film and patterned lines, resulting from the measurement 6 months after the annealing. As expected, the straight lines from both measurements on the lines intersect at $\sin ^{2} \Psi=0$. The slight scatter in the low- $\Psi$ region is the main reason for typical errors of $10 \mathrm{MPa}$ (films) and $20 \mathrm{MPa}$ (lines) in the calculated stresses.

The measured values $d_{0}$ for the unstressed lattice spacing depend on the adjustment of the diffractometer and possibly on the microstructure of the samples. An uncertainty in $d_{0}$ of $0.0001 \AA$ causes a change of the stress component for lines amounting to about $30 \mathrm{MPa}$. Therefore $d_{0}$ was interpolated for each sample from the continuous film measurements. We found $d_{0}$ to amount to $0.82655,0.82647$, 0.82660 , and $0.82652 \AA$ for unpassivated AL, passivated $\mathrm{AL}$, unpassivated II, and passivated II, respectively. In both $\mathrm{AL}$ and II films passivation results in a change of $d_{0}$, which may be attributed to the additional thermal cycle during the passivation process. Using these $d_{0}$ values, strains were obtained which are listed in Table I.

Continuous films show tensile strain in the film plane and compressive strain perpendicular to the film surface, as expected for films that are free to contract in the $z$ direction. Passivation leads to a slight increase in ion-implanted films. Surprisingly, AL films exhibited somewhat smaller strains after passivation-an effect which is presently not fully understood. Relaxation resulted in a systematic reduction of strains by about $30 \%$ (AL) and $60 \%$ (II).

The unpassivated lines show tensile strain in the length direction, small compressive strain in the width and bigger compressive strain along the height direction. Like continuous films, unpassivated lines are free to contract in $z$ direc- tion, leading to zero stress in this direction. The constraining influence of the substrate due to the different thermal expansion coefficients is more distinct in the $x$ direction than in the $y$ direction. This stands to reason as unpassivated lines are able to shrink in width, at least at some distance from the substrate. ${ }^{6}$ This is no longer true for passivated lines because passivation suppresses free contraction in all three directions. Here, like in the $x$ direction the line is under tensile strain also in the $y$ direction and $\epsilon_{y}$ can nearly reach the same value as $\epsilon_{x}$.

From the strains, stresses were calculated as described above. The results are compiled in Table II together with the calculated hydrostatic and Tresca maximum shear stresses in the lines. In the case of unpassivated continuous films, the stresses after annealing correspond reasonably well to those reported in Ref. 5 (AL:280 MPa,II: $440 \mathrm{MPa}$ ), measured with the wafer curvature method. A comparison of the amount of stress relaxation in AL films $(-30 \%)$ with II films $(\sim 50 \%-60 \%)$ shows that more relaxation takes place in ionimplanted films during the same time, i.e., relaxation is faster. This may be explained with the smaller grain size of the ion-implanted films, ${ }^{7}$ which enhances the contribution of stress-driven grain-boundary diffusion (Coble creep) to the relaxation process.

Next we discuss the case of unpassivated lines. Here, the stress in the length direction is nearly of the same amount as in the corresponding continuous films, if the relaxed values are considered. This was to be expected because in this direction no geometric change has taken place during patterning. The stress across the width of the lines is much smaller than in the length direction because of the reduced constraint as discusscd above. For the same reason $\sigma_{y}$ is much smaller

TABLE II. Summary of the measured stresses (in MPa) in continuous films and patterned lines ( $\sigma$-biaxial stress, $\tau-$ maximum shear stress).

\begin{tabular}{|c|c|c|c|c|c|c|c|}
\hline & & \multirow{2}{*}{$\frac{\text { Films }}{\sigma}$} & \multicolumn{5}{|c|}{ Lines } \\
\hline \multicolumn{2}{|c|}{ Sample description } & & $\sigma_{x}$ & $\sigma_{y}$ & $\sigma_{z}$ & $\sigma_{\text {hydr }}$ & $\tau$ \\
\hline AL & as annealed & 200 & 330 & 100 & 0 & 145 & 165 \\
\hline (unpassivated) & relaxed & 140 & 120 & 30 & 0 & 50 & 60 \\
\hline $\mathrm{AL}$ & as annealed & 160 & 570 & 535 & 200 & 435 & 185 \\
\hline (passivated) & relaxed & 105 & 380 & 300 & 220 & 300 & 80 \\
\hline II & as annealed & 440 & 240 & 90 & 0 & 110 & 120 \\
\hline (unpassivated) & relaxed & 170 & 170 & 55 & 0 & 75 & 85 \\
\hline II & as annealed & 470 & 415 & 330 & 170 & 305 & 122 \\
\hline (passivated) & relaxed & 250 & 200 & 190 & 170 & 185 & 15 \\
\hline
\end{tabular}


TABLE III. Summary of the measured stresses (in MPa) in passivated lines and those calculated from the simple addivity rule, Eqs. (1) and (2).

\begin{tabular}{|c|c|c|c|c|c|c|c|}
\hline & & \multicolumn{3}{|c|}{ Measured values } & \multicolumn{3}{|c|}{ Calculated values } \\
\hline \multicolumn{2}{|c|}{ Sample description } & $\sigma_{x}$ & $\sigma_{y}$ & $\sigma_{z}$ & $\sigma_{x}$ & $\sigma_{y}$. & $v_{z}$ \\
\hline $\mathrm{AL}$ & as annealed & 570 & 535 & 200 & 530 & 300 & 200 \\
\hline (passivated) & relaxed & 380 & 300 & 220 & 340 & 250 & 220 \\
\hline II & as annealed & 415 & 330 & 170 & 410 & 260 & 170 \\
\hline (passivated) & relaxed & 200 & 190 & 170 & 340 & 225 & 170 \\
\hline
\end{tabular}

than $\sigma_{x}$ in the as annealed unpassivated lines. But in this case $\sigma_{x}$ is not comparable to the continuous film value. For AL, $\sigma_{x}$ is higher than in the continuous film and corresponds to the wafer curvature value. For II, $\sigma_{x}$ is only nearly half of the film stress. A possible explanation is the longer measuring time required for the line measurements compared to the continuous film measurement. Due to the fast relaxation it is possible that the measured stresses do not correspond to the real as annealed but to some relaxed state.

Passivation has a different effect on films and lines. Passivated films show similar stress levels as the equivalent unpassivated films, i.e., a passivation layer has nearly no effect on stress because the film is still free to deform in the $z$ direction. This changes in the case of passivated patterned lines. Now the dilatation is restricted in all three directions leading to a triaxial state of stress. In the as-annealed state high stresses, especially along the lines, were measured. These values, which amount to 570 and $415 \mathrm{MPa}$ for pure and ion-implanted aluminum, respectively, can be regarded as a lower bound to the yield strength of the material when surrounded by a stiff matrix. These are very high values compared to bulk material. The reason is that in such small dimensions geometrical restrictions play an important role for dislocation slip mechanisms and consequently for processes like plastic deformation (small scale effects).

Like on unpassivated lines, all measurements with passivated lines show that $\sigma_{x}>\sigma_{y}>\sigma_{z}$. This results from the fact that the substrate mostly influences the contraction in $x$ direction and not at all in $z$ direction, where it only is hindered when a passivation layer is present. After 6 months of relaxation the three stress components of the II lines are nearly the same, i.e., the stress is approximately hydrostatic. This development is also reflected in the decrease of the maximum shear stress, which is particularly obvious in the case of the II lines with passivation. The low shear stress in that case indicates that the attempt to mechanically strengthen the line by ion implantation has failed completely. There are two possible reasons for this: First, as discussed above for films, the small grain size of the II lines offers a higher grain boundary density as diffusion paths for Coble creep-type relaxation processes; by contrast, the Al lines exhibited near-bamboo grain structures which slow or suppress grain-boundary relaxation. Second, the second-phase particles present in the II lines may have acted as convenient void nucleation sites, especially under the hydrostatic stress state in passivated lines. This explanation is also consistent with the observation in Table II that ion implantation tends to lower the stress values in lines while it increases the stresses in films.

Finally, we note that the effect of passivation on the stress components seems to follow a simple rule. Assuming that the only difference of the stresses in unpassivated and passivated lines of the same material arises from an addition of a hydrostatic stress, $\sigma_{z}$ of passivated lines might be regarded as the hydrostatic stress value of the passivation. The sums of this value and the measured stress components of unpassivated lines according to

$$
\sigma_{x}^{\text {pass }}=\sigma_{x}^{\text {unpass }}+\sigma_{z}^{\text {pass }}
$$

and

$$
\sigma_{y}^{\text {pass }}=\sigma_{y}^{\text {unpass }}+\sigma_{z}^{\text {pass }}
$$

can be compared with the measured stress components of the passivated lines. Table III shows that the agreement is very good for $\sigma_{x}$ for AL (both measurements) and for the II measurement in the as-annealed state, whereas the relaxed value differs considerably. This can again be attributed to the small grain size that renders possible the development of a purely hydrostatic state of stress. For the $y$ direction the agreement is not as good, as might have been expected because of the shrinkage in the width direction of unpassivated lines discussed above.

The present results, if substantiated, may have important consequences for conductor line materials. The hope that the electromigration resistance can be improved by mechanical strengthening due to second phase particles ${ }^{3}$ may not materialize if the particles allow easy nucleation of pores under hydrostatic stresses. Further work along this line is currently in progress.

${ }^{1}$ B. Greenebaum, A. I. Sauter, P. A. Flinn, and W. D. Nix, Appl. Phys. Lett. 58, 1845 (1991)

${ }^{2}$ J. W. McPherson and C. F. Dunn, J. Vac. Sci. Technol. B 5, 1321 (1987).

${ }^{3}$ E. Arzt and W. D. Nix, J. Mater. Res. 6, 731 (1991).

${ }^{-4}$ P. A. Flinn and C. Chiang, J. Appl. Phys. 67, 2927 (1990).

${ }^{5}$ E. Arzt, O. Kraft, J. Sanchez, S. Bader, and W. D. Nix, Mater. Res. Soc. Symp. Proc. 239, 677 (1991).

${ }^{6}$ A. I. Sauter, Ph.D. thesis, Stanford University, 1991.

${ }^{7}$ S. Bader, P. A. Flinn, E. Arzt, and W. D. Nix, J. Mater. Res. 2, 9 (1994). 